\title{
Introduction: Hubert Aquin et les médias
}

\section{Gilles Dupuis}

Université de Montréal

François Harvey

Collège Édouard-Montpetit

Hubert Aquin est sans aucun doute une des figures les plus importantes du paysage culturel québécois. Ses écrits romanesques, novellistiques, et essayistiques, abondamment diffusés grâce à l'Édition critique de l'œuvre d'Hubert Aquin (ÉDAQ), ont fait l'objet d'innombrables études qui ont contribué à la reconnaissance et à la consécration de l'auteur. Il en va cependant autrement pour les radiothéâtres, les téléthéâtres, et les films scénarisés, réalisés et produits par Aquin. Pour la plupart inédits, ils ont rarement été pris en considération par la critique aquinienne, à l'exception de quelques articles et de l'étude pionnière Hubert Aquin et la radio. Une quête d'écriture (1954-1977) de la spécialiste des médias Renée Legris. Pourtant, parallèlement à ses activités littéraires et politiques, l'écrivain québécois a œuvré longuement dans les médias, tant à la télévision et à la radio qu'au grand écran.

Aquin amorce sa carrière médiatique lors de son séjour d'étude en Europe au début des années 1950. ${ }^{1}$ Ayant obtenu une bourse du gouvernement français, puis du ministère du Bien-être social et de la Jeunesse à Québec, il part pour la France à l'automne 1951 et s'inscrit à l'Institut d'études politiques à Paris, puis, en 1952, à la faculté de lettres de la Sorbonne où il projette d'écrire une thèse d'inspiration phénoménologique sur la création du personnage romanesque. En marge de ses études, il accepte quelques contrats au Service canadien de la RTF (Radiodiffusion-télévision française) où il est affecté au mixage de certaines émissions et où il travaille occasionnellement en tant que comédien et chroniqueur culturel. Dans ses échanges épistolaires avec sa famille, Aquin fait fréquemment référence à ces contrats 
radiophoniques, annonçant par exemple la diffusion prochaine sur les réseaux canadiens de ses chroniques de voyages (à l'antenne de CKAC ou de Radio-Canada), inspirées de ses séjours en Suisse et en Grèce: "Il y a deux jours j’ai enregistré quatre émissions dont une sur la Suisse et trois sur la Grèce - mais elles ne passeront que vers la fin de novembre sur les ondes canadiennes" (Aquin 1952). Il évoque également le petit rôle qu'il tient dans un radiothéâtre composé par son ami Jacques Languirand:

Ces jours-ci il m'est arrivé quelque chose de vraiment nouveau: je suis interprète dans une émission de la Radiodiffusion française; cette émission est un programme de Noël qui passera sur les ondes de RadioCanada, dans les quelques jours qui précéderont le jour de Noël; j’y joue deux petits rôles pas très importants, une vingtaine de répliques au plus, et le tout me paiera environ six dollars. Pour vous aider à identifier l'émission: le texte est de Jacques Languirand, la vedette Lucienne Letondal, durée: 30 minutes. C'est demain soir que nous enregistrons. (Aquin 1951, nous corrigeons les coquilles)

Rentré au Québec le 10 mai 1954, Aquin postule pour un emploi à la Société Radio-Canada où travaille déjà son ami et complice LouisGeorges Carrier. Il est embauché au rang de stagiaire le $1^{\text {er }}$ juillet 1954. Pendant deux mois, il fait ses classes à la radio, sous la tutelle du réalisateur Guy Mauffette. Son stage est une expérience formatrice décisive pour sa carrière médiatique; il en dira, dans une lettre à Pierre Pagé:

Je n'oublierai jamais les instants délirants de mon apprentissage. Mon maître m'a d'abord ébloui par ses apparitions fulgurantes, ses farces à répétition, sa théâtralité récurrente, son tonus increvable. J'ai été subjugué par la grande maitrise dont Guy Mauffette faisait preuve dans l'utilisation des disques: chansons-nouveautés, transitions musicales, ainsi qu'au micro - comme disque-jockey. (Legris et Pagé 307)

Le 27 juillet 1954, Aquin est informé de sa nomination à un poste de réalisateur à la radio, fonction qui sera confirmée le 7 septembre suivant. De l'année de son embauche à celle de sa démission en 1959, il remplit plusieurs fonctions au sein la société d'État, dont les titres et les rôles ne sont pas toujours bien définis. D'abord réalisateur, Aquin obtient au début de 1956 le titre d'organisateur d'émissions 
(ou organisateur des programmes). Bien qu'il continue ses activités de réalisation, il se voit attribuer de nouvelles tâches qui tiennent de la supervision et de l'organisation d'émissions télévisuelles. Au début de 1957, il obtient une promotion et devient coordonnateur des émissions éducatives à la télévision puis, au cours de l'été 1957, cadre hors statut, titre temporaire le menant, le 17 décembre 1957, à celui de superviseur (Supervising Producer), dont la fonction consiste en la "coordination des équipes de réalisation, [...] à la mise en place des programmes et à l'évaluation de leur qualité" (Legris 36). En avril 1958, il est à nouveau promu et devient le directeur adjoint de Marc Thibaut, directeur du Service des émissions éducatives et d'affaires publiques (SÉÉAP), en plus de remplir les tâches de coordonnateur de programmes éducatifs et culturels à la télévision et à la radio. Pendant les cinq années passées à l'emploi de Radio-Canada, Aquin gravit les échelons de la société d'État, jusqu'au 16 octobre 1959, date à laquelle il remet sa démission afin de passer à l'Office national du film (ONF).

Parallèlement à ses tâches de réalisateur et de coordonateur à la Société d'État, Aquin rédige plusieurs documentaires pour la radio dont trois émissions portant sur des figures majeures de la littérature, diffusées dans le cadre de la série Les Hommes illustres en 1954 et 1955: "Pascal," "Dostoïevski," et "André Malraux." En plus d'adapter trois œuvres littéraires pour la radio et la télévision, soit L'Homme qui a perdu son ombre de Paul Gilson (radio, 1954), Moïra de Julien Green (télévision, 1955; la pièce fit scandale lors de sa diffusion), et L'Échange de Paul Claudel (télévision, 1960), ${ }^{2}$ Aquin compose plusieurs dramatiques originales, dont La Toile d'araignée (radio, 1954), Histoire d'amour (télévision, 1955; cette pièce a apparemment été refusée par Radio-Canada), Passé antérieur (télévision, 1955; le texte de cette dramatique est perdu), Le Choix des armes (télévision, 1959; cette dramatique rédigée en 1958 n'a jamais été diffusée en raison de la grève des réalisateurs de la Société Radio-Canada), Dernier acte (télévision, 1960), et Oraison funèbre (télévision, 1962; la rédaction de cette dramatique a débuté à la fin de 1958 sous le titre de L'Efficacité). Les années passées à travailler pour la Société Radio-Canada ont été riches d'enseignements pour Aquin qui, en plus d'avoir composé une œuvre documentaire et dramatique foisonnante, a acquis les codes d'écriture et les mécanismes de production spécifiques aux médias radiophonique et télévisuel, essentiels pour son passage à l'ONF. 
Aquin entre officiellement à l'Office national du film au mois de novembre 1959. Il remplit au sein de l'agence culturelle du gouvernement fédéral plusieurs rôles, dont ceux de scénariste, de réalisateur, et de producteur. En tant que scénariste, il compose les textes d'À l'heure de la décolonisation (1963) et il participe à l'élaboration de La Fin des étés (1964) d'Anne-Claire Poirier. Réalisateur, il met en scène trois films d'importance: Le Sport et les hommes (1961), dont Roland Barthes fournit le commentaire sur le modèle des Mythologies; Le Temps des amours (1961), série de films destinés à la télévision portant sur les relations amoureuses dans différents pays, pour laquelle Aquin écrit et réalise la version française de l'épisode canadien; et A Saint-Henri le 5 septembre (1962), film tourné, comme son titre l'indique, le 5 septembre 1961 dans le quartier populaire francophone de Montréal. Crédité de la réalisation d'À Saint-Henri le 5 septembre, il s'avère en réalité que l'apport d'Aquin à cette œuvre emblématique du cinéma direct est mince, voire nébuleux. Dès le 5 juillet 1961, dans son journal, l'écrivain émet des réserves sur sa participation à la réalisation de ce film et, le jour suivant, il mentionne avoir "renoncé à 'Saint-Henri' pour ne pas compromettre [un] voyage en Europe" (Aquin 1992, 204) qu'il effectuera pour le compte de l'ONF et qui le mènera jusqu'en Afrique. Comme le rappelait récemment Jacques Godbout, Aquin n'aurait donc participé qu'à la phase préparatoire du documentaire:

La réalisation du 5 septembre, attribuée dans les documents de l'ONF à mon ami Hubert Aquin, chargé de projet, tient à un quiproquo. L'ami Hubert était un spécialiste des rendez-vous manqués. C'est ainsi qu'il n'était ni au tournage ni au visionnement des "rushes," et encore moins à celui du montage. En réalité, Aquin était évanescent, il se plaisait à dire "je déçois" et s'était lancé dans un projet de course automobile sur l'île Sainte-Hélène. Pendant que je rédigeais la narration de Saint-Henri, Hubert Aquin dînait à Rome avec Fangio, discutant de l'avenir de la Formule 1 à Montréal. À l'époque, les crédits cinématographiques comptaient pour peu et en lui accordant la "réalisation" du 5 septembre à Saint-Henri, nous lui faisions nos amitiés. Il quittait l'ONF peu après. $(33-34)$

Les tâches de réalisateur et de producteur à l'ONF portent Aquin à effectuer plusieurs voyages au Canada et à l'étranger. À l'automne 1960, 
il se rend à Paris pour un séjour d'un mois afin d'y rencontrer Roland Barthes, avec lequel il travaille à l'élaboration du documentaire Le Sport et les hommes. En novembre 1961, il visite plusieurs régions d'Afrique, dont le Sénégal, le Dahomey (l'actuel Bénin), et la Côte-d'Ivoire, où il prépare la série Civilisation française, pilotée par Fernand Dansereau, qui comprend initialement six films ayant "pour thème la diffusion de la culture française à travers le monde" (Massoutre 132), et dans le cadre de laquelle sera réalisé le documentaire $A$ l'heure de la décolonisation. Aquin retourne en Europe en juin 1962 afin de continuer son travail sur Civilisation française. Concentrant ses énergies sur $\grave{A}$ l'heure de la décolonisation, il interviewe plusieurs intellectuels à Paris, dont Albert Memmi et Paul Ricœur, puis il se rend à Lausanne où il tourne des entrevues avec le Front de libération nationale algérien (FLN). Il profite de son passage dans la capitale du canton de Vaud pour rencontrer l'écrivain Georges Simenon, avec lequel il s'entretient durant trois jours; consignant ses interviews sur pellicule, il projette de réaliser un documentaire sur le romancier, mais le film ne sera jamais monté. ${ }^{3}$ De retour à Paris, Aquin s'envole enfin pour l'Italie en juillet 1962 afin de préparer le tournage de L'Homme vite (1963), film dont il est le producteur et dans lequel il "se propose de mettre en lumière le combat de l'homme contre le temps que le coureur automobile exprime dans son rapport à la vitesse" (Massoutre 131). De Rome, il projette de se rendre à Modène et à Turin, mais son fils Philippe, “dans un état grave à l'hôpital," (Aquin 1992, 241) le rappelle au pays.

Le manque d'organisation et les dépenses excessives d'Aquin lui valent d'être réprimandé par Fernand Dansereau. Pressentant son congédiement, comme il le rapporte dans son journal à l'entrée du 15 juillet 1962 - "Un dénouement désastreux menace de s'abattre sur moi à l'ONF: congédiement, ou quelque chose d'approchant!" (Aquin 1992, 242) -, il se voit retirer la production de la série Civilisation française et est rétrogradé au rang de réalisateur. Contrarié par sa condamnation "à ne retirer aucun crédit du succès éventuel de cette série" (Aquin 1992, 369), Aquin n'en demeure pas moins convaincu de la pertinence de sa carrière cinématographique, comme il le soutient dans une lettre à Grant McLean, directeur de la production à l'ONF:

[J]e tiens à dire que je suis entré à l'ONF, en 1959, pour faire du cinéma. J'en ai fait avec enthousiasme et sincérité. Le mémoire de 
Fernand Dansereau m'oblige à faire des preuves que je croyais avoir déjà fournies. Je suis prêt à en fournir de nouvelles puisque de toute façon en continuant à travailler à "Civilisation française" je n'aurais pu faire autrement qu'en produire, sans toutefois qu'on m'y contraigne. Je demeure profondément intéressé à faire des films et à ne pas gaspiller ceux-ci sous prétexte de faire leur procès avant même qu'ils existent. (Aquin 1992, 370)

Bien qu'Aquin demeure à l'emploi de l'Office national du film jusqu'en 1964, les grands projets cinématographiques sont désormais derrière lui. Les intérêts de l'écrivain se portent plutôt vers l'action politicolittéraire: terroriste autoproclamé, il entre dans la clandestinité en juin 1964. Arrêté puis interné, il écrit son premier roman Prochain épisode entre les murs de l'Institut Prévost et le publie en novembre de l'année suivante. Sa carrière de romancier débute alors avec fracas.

L'entrée officielle d'Aquin en littérature ne freine pas sa carrière médiatique. Pigiste pour la Société Radio-Canada depuis 1959, il collabore régulièrement avec la société d'État jusqu'à sa mort en 1977. Adaptateur, traducteur, documentariste, et scénariste, il rédige une foule d'émissions radiophoniques et télévisuelles. Pour la radio, il compose son second radiothéâtre au début des années 1960, Confession d'un Héros (1961), puis de nombreuses émissions documentaires portant sur des sujets aussi divers que l'histoire politique des Amériques, la littérature, la religion, et la philosophie, notamment les textes "Saint Augustin" et "Nietzsche" pour la série Philosophes et penseurs (1963-1964). Pour la télévision, Aquin traduit et adapte plusieurs textes dramatiques, dont Yerma de Federico Garcia Lorca (1961), La Pie-grièche de Joseph Kramm (The Shrike, 1963), La Parisienne d'Henri Becque (1966), Faux bond de Jean-Charles Tacchella (1967; Aquin y tient le rôle principal de l'agent triple Hubert Desaulniers), et Une femme en bleu au fond d'un jardin de pluie de George Ryga (Sunrise on Sarah, 1974). Les années 1960 et 1970 sont également fécondes sur le plan de l'écriture dramatique, l'écrivain ayant composé ses téléthéâtres les plus achevés au cours de cette période: Table tournante (1968), 24 heures de trop (1969), et Double sens (1972). En plus des œuvres diffusées à la radio et à la télévision, Aquin a également élaboré de multiples projets télévisuels et filmiques, parmi lesquels figurent Faites-le vous-même, “dramorama" coécrit avec Louis-Georges Carrier entre 1967 et 1970, Histoire de l'anarchie, film élaboré avec le concours de Jacques Godbout 
au début des années 1970, et une adaptation d'Edipe de Sophocle, dont l'écriture s'est étendue sur deux ans (1971 et 1972). La carrière médiatique d'Aquin prend véritablement fin avec la rédaction de "Nabokov ou l'exil," documentaire radiophonique portant sur l'auteur de Lolita composé avec le concours de Pierre Turgeon et diffusé posthumément, le 4 octobre 1977. Riche de réalisations diverses, les créations radiophoniques et écraniques d'Aquin ont constitué une part importante de son parcours intellectuel où se sont élaborées certaines des grandes obsessions de son œuvre essayistique et littéraire, comme le nationalisme, le dédoublement identitaire, et le suicide.

La carrière radiophonique, télévisuelle, et filmique d'Hubert Aquin, dont nous avons rapidement esquissé les contours dans cette brève introduction, restait donc à explorer. C'est ce que nous nous sommes proposé de faire dans ce numéro spécial de Québec Studies, à travers l'analyse de certains éléments les plus significatifs de l'œuvre médiatique aquinienne. Par souci historique, nous avons classé les articles qui composent notre dossier dans l'ordre chronologique où se sont manifestés les intérêts d'Aquin envers les médias radiophonique, télévisuel, et filmique qu'il a tour à tour explorés. Nous entamons notre incursion par la présentation d'un inédit radiophonique d'Aquin, le scénario du documentaire "Nietzsche," diffusé le 24 mai 1964 sur les ondes de Radio-Canada dans le cadre de la série Philosophes et penseurs. Dans une langue stylisée et poétique, Aquin y recense les événements les plus pertinents de la vie du philosophe, tout en exposant les grands thèmes de sa pensée, notamment ceux qui se rapportent à la mort de Dieu et à la volonté de puissance. Le scénario d'Aquin est suivi de trois articles portant sur la carrière télévisuelle aquinienne. Dans "Les CEdipe d'Aquin: écriture (dialoguée, inachevée) médiatique à quatre mains," Renald Bérubé s'intéresse au projet d'adaptation d'CEdipe, analysant les rapports, parfois conflictuels, qu'ont entretenus Hubert Aquin et Louis-Georges Carrier en vue de la réalisation de cette dramatique inachevée. François Harvey se penche ensuite sur le concept de simulacre dans le dernier téléthéâtre original composé par Aquin, Double sens, dont il observe à la fois les articulations dans le scénario de l'écrivain et dans l'adaptation télévisuelle qu'en a tirée Louis-Georges Carrier. L'article d'Harvey est suivi d'un texte de Renée Legris intitulé "Le Temps des amours. Hubert Aquin, auteur et réalisateur d'un film pour la télévision" où la spécialiste de l'œuvre 
médiatique aquinienne s'intéresse à la genèse, aux conditions de réalisation et aux thèmes de cette émission télévisuelle produite par l'ONF, dont Aquin a réalisé le quatrième épisode qui traite des mœurs amoureuses des jeunes Canadiens. La dernière partie de ce dossier est consacrée au cinéma d'Aquin. Dans "L'essai cinématographique chez Hubert Aquin," Vincent Bouchard effectue une comparaison entre Le Sport et les hommes (1961) et deux films phares du cinéma documentaire des années 1960, La Lutte (Michel Brault, Marcel Carrière, Claude Fournier, et Claude Jutra, 1961) et Vive le Tour! (Louis Malle, 1962). Pour sa part, Gilles Dupuis s'intéresse au motif du faux dans l'œuvre littéraire et filmique d'Aquin, prenant comme point d'appui de son analyse critique le fameux film d'Orson Welles, F for Fake.

Les études qui composent le présent dossier ont pour ambition d'offrir de nouvelles perspectives d'analyse de l'œuvre d'Hubert Aquin, tout en comblant une lacune importante dans la critique aquinienne qui a souvent négligé, voire trop longtemps ignoré un pan entier de cette œuvre représenté par la production médiatique, pourtant révélatrice des desseins politiques et poétiques, éthiques, et esthétiques que poursuivait l'auteur. S'inscrivant dans la foulée des travaux de Danielle Aubry, de Renée Legris, et de Pierre Pagé sur les médias radiophonique et télévisuel, ce dossier vise aussi à élargir le champ des études médiatiques québécoises qui, contrairement à ce qui a cours dans les mondes anglo-saxon, américain, et européen, reste encore aujourd'hui largement inexploré.

\section{Notes}

1 Ces éléments biographiques prennent leur source dans les travaux de Guylaine Massoutre, Itinéraires d'Hubert Aquin et "Hubert Aquin à Radio-Canada. Enquête bio-bibliographique. Étude des sources" (en collaboration avec Denis Desjardins). Nous avons complété nos observations au moyen de l'ouvrage de Renée Legris, Hubert Aquin et la radio. Une quête d'écriture (1954-1977), où sont apportées quelques corrections aux éléments relevés par Massoutre, et par nos propres recherches dans les archives de la Société Radio-Canada, de l'Université du Québec à Montréal, et du Dépôt électronique et vitrine de consultation des archives en littérature et culture québécoises de l'Université Laval (DÉCALCQ).

2 Dans son ouvrage Hubert Aquin et la radio. Une quête d'écriture (1954-1977), Renée Legris suggère qu'Aquin aurait adapté pour la radio au cours de l'année 1954 le conte La Légende de l'homme à la cervelle d'or d'Alphonse Daudet et quatre épisodes de la série La Chronique des Pasquiers inspirée de l'œuvre de Georges Duhamel (43). Il 
appert cependant que, selon les données que nous avons recueillies dans les archives radio-canadiennes et dans La Semaine à Radio-Canada, l'écrivain n'aurait participé qu'à la réalisation du conte de Daudet dont l'adaptation a été confiée à Marcelle Bazzana et qu'il n'aurait composé aucun épisode de La Chronique des Pasquiers dont la charge incombait plutôt à l'actrice Françoise Faucher. Soulignons également que selon Legris, Aquin aurait participé en 1962 à la rédaction d'une série radiophonique intitulée Sur quatre roues et diffusée à l'antenne de Radio-Canada à l'été 1963 (169). Cette information figure également dans le Répertoire Hubert Aquin. Bibliographie analytique 1947-1997 de Manon Dumais et Jacinthe Martel - qui situent toutefois la diffusion de cette série à l'été 1962 (308). Nos recherches au sujet de Sur quatre roues n'ont pu déterminer de manière exacte l'apport d'Aquin à cette série.

3 Selon Guylaine Massoutre, le matériel tourné lors de ces entrevues a été égaré (Massoutre 1992, 134).

\section{Ouvrages cités et consultés}

Aquin, Hubert. "Lettre d'Hubert Aquin à ses parents, Paris, dimanche le 28 octobre 1951.” Fonds Hubert-Aquin (Philippe et Stéphane Aquin), Service des archives et de gestion des documents de l’Université du Québec à Montréal, 1951.

—. "Lettre d'Hubert Aquin à ses parents, Paris, 25 octobre 1952." Fonds HubertAquin (Philippe et Stéphane Aquin), Service des archives et de gestion des documents de l'Université du Québec à Montréal, 1952.

- Journal 1948-1971. Édition critique établie par Bernard Beugnot, Montréal: Bibliothèque québécoise, 1992.

- Trou de mémoire. Édition critique établie par Janet M. Paterson et Marilyn Randall, Montréal: Bibliothèque québécoise, 1993.

- Prochain épisode. Édition critique établie par Jacques Allard, Montréal: Bibliothèque québécoise, 1995.

- Neige noire. Édition critique établie par Pierre-Yves Mocquais, Montréal: Bibliothèque québécoise, 1997.

Dumais, Manon et Jacinthe Martel. Répertoire Hubert Aquin. Bibliographie analytique 1947-1997. Montréal: Université du Québec à Montréal, Département d'études littéraires, 1998.

Godbout, Jacques. "Lettre ouverte à Shannon Walsh, à propos de Saint-Henri." Spirale 238 (automne 2011): 33-34.

Lahaie, Christiane. "Hubert Aquin ou la quête médiatique." Fréquence 3-4 (1995): 87-100.

Legris, Renée. Hubert Aquin et la radio. Une quête d'écriture (1954-1977). Montréal: Médiaspaul, 2004.

Legris, Renée et Pierre Pagé. "Le théâtre à la radio et à la télévision au Québec." Le Théâtre canadien-français. Évolution. Témoignages. Bibliographie. Montréal: Fides, coll. "Archives des lettres canadiennes," 1976. 291-318.

Massoutre, Guylaine. Itinéraires d'Hubert Aquin. Montréal: Bibliothèque québécoise, 1992. 
Massoutre, Guylaine et Denis Desjardins. "Hubert Aquin à Radio-Canada. Enquête bio-bibliographique. Étude des sources.” Bulletin de l'ÉDAQ 5 (décembre 1985): $17-31$.

\section{Médiagraphie des œuvres radiophoniques, télévisuelles et filmiques}

\section{Radio}

Documentaires:

— Série Les Hommes illustres (1954-1955): "Pascal," "Dostoïevski," “André Malraux"

- Série L'Homme américain (1963): "L'invention de l'Amérique latine," "Première rupture," "Le Canada de 1840," “Cuba est une île," “L'homo politicus américain"

— Série Philosophes et penseurs (1963-1964): "Introduction à Moïse," "Moïse," "Saint Paul," "Saint Augustin," "Mahomet," "Nietzsche," "Henri Bergson," "Freud," "Jean-Paul Sartre"

- Série Documents: "Don Quichotte, le héros tragique," (1966) "Paul-Émile Borduas et le Refus global" (1968)

— Hors série: "Nabokov ou l'exil" (en collaboration avec Pierre Turgeon, 1977)

Radiothéâtres:

- La Toile d'araignée (1954)

- Confession d'un héros (1961)

Adaptations:

- L'Homme qui a perdu son ombre de Paul Gilson (1954)

- Célébration du vin de Maurice Lelong (1968)

- Célébration du corps de François Solomon (nos recherches dans La Semaine à Radio-Canada ne nous ont pas permis de confirmer la diffusion de ce texte qui, selon Manon Dumais, Jacinthe Martel (88), et Renée Legris (43), aurait été mis en ondes le 9 octobre 1968)

- Célébration de la pomme de terre de Jean Follain (1968)

Autres:

- De retour le 11 avril... (nouvelle diffusée dans le cadre de l'émission "Nouvelles inédites," 1968)

\section{Télévision}

Cuvres originales:

- Passé antérieur (1955)

- Le Choix des armes (1959)

- Dernier acte (téléthéâtre écrit sous le pseudonyme de François Lemal, 1960)

- On ne meurt qu'une fois (minisérie coécrite avec Gilles Sainte-Marie, 1960)

- Oraison funèbre ou L'Efficacité (1962)

- Table tournante (1968)

- 24 heures de trop (1969)

- Double sens (1972) 
Adaptations:

- Moïra de Julien Green (1955)

- L'Échange de Paul Claudel (1960)

- Yerma de Federico Garcia Lorca (1961)

- La Pie-grièche de Joseph Kramm (1963)

- La Parisienne d'Henri Becque (1966)

- Faux bond de Jean-Charles Tacchella (1967)

- Une femme en bleu au fond d'un jardin de pluie de George Ryga (1974).

Projets de téléthéâtres:

- Histoire d'amour (1955)

- Jules César ou La Mort de César (1960-1962)

- Faites-le vous-même ou Smash (1967-1970)

- Ulysse (1970)

- Hamlet (1970)

- Le Mal (1970)

- Opération labyrinthe (1970-1971)

- Edipe et Edipe recommencé (1971-1972)

- La Mort de Charité (1973)

- Les Plaisirs de la mort ou Je suis mort ou L'Invention de la mort (1974)

\section{Cinéma}

Scénariste:

- À l'heure de la décolonisation (texte d'Hubert Aquin, 1963)

- La Fin des étés (scénario d'Hubert Aquin et d'Anne-Claire Poirier, 1964)

Réalisateur:

- Le Sport et les hommes (réalisation d'Hubert Aquin, 1961)

- Le Temps des amours (réalisation, scénario et production d'Hubert Aquin, 1961)

- À Saint-Henri le 5 septembre (réalisation attribuée à Hubert Aquin, 1962)

Producteur:

- Quatre enfants du monde (production d'Hubert Aquin et de Ian MacNeill, 1959)

- L'Exil en banlieue (production d'Hubert Aquin, 1960)

- Les Grandes religions (production d'Hubert Aquin, 1960)

- Quatre instituteurs (production d'Hubert Aquin, de Julian Biggs, et de Guy Glover, 1961)

- Jour après jour (production d'Hubert Aquin, de Fernand Dansereau, et de Victor Jobin, 1962)

- L'Homme vite (production d'Hubert Aquin, 1963)

- Jour de mariage (production d'Hubert Aquin et de Gordon Burwash, 1963)

- Trois pays, trois grand-mères (production d'Hubert Aquin, de Gordon Burwash, et de Guy Glover, 1963)

- Trois pays, trois apprentis (production d'Hubert Aquin et de Gordon Burwash, 1963) 
- Trois pays, trois artistes (production d'Hubert Aquin, de Gordon Burwash, et de Guy Glover, 1964)

- Trois pêcheurs (production d'Hubert Aquin, de Julian Biggs, et de Guy Glover, 1964)

Projets de films:

- Projet de film expérimental (date inconnue)

- Histoire de l'anarchie (en collaboration avec Jacques Godbout, 1970-1971)

- La Reine de Pologne ou Sacrilège (1976) 